\title{
An efficient wide-band signal detection and extraction method
}

\author{
Qian Cheng, Kexian Gong ${ }^{*}$, Min Zhang, and Xiaoyan Liu
}

Electronics and Communication Engineering, College of Information Engineering, Zhengzhou University, 100 Science Avenue,Zhengzhou City, Henan Prov, China

\begin{abstract}
Aiming at the influence of time-varying and frequency-varying of noise on the signal detection performance in the short wave wide-band channel and the large amount of computation in the channelized receiver model of the traditional low pass filter bank, a cross-channel reconfigurable multi-phase high-efficiency channelization method based on morphological processing is proposed in this paper .Firstly, The wide-band signal is coarsely filtered by the multi-phase structure of the uniform filter bank which is determined by the protection interval between signals, and then the bandwidth and position of the signal are determined by improved morphological operation and threshold decision of the power spectrum. Finally, the sub-band signals across the channel are combined to complete the approximate reconstruction of the sub-signals. Compared with the computational complexity of traditional channelized receiver model, the results show that this method has lower computational complexity. The simulation results show that the method can achieve the approximate constant false alarm rate(CFAR) under the colored noise environment, and has higher detection capability under different signal-to-noise ratios(SNR).
\end{abstract}

\section{Introduction}

The acquisition bandwidth of short-wave narrowband receiver is generally $3 \mathrm{Khz}$. The increasingly advanced short-wave communication technology has got rid of the traditional detection technology [1].

Due to the increasing communication demand, the communication environment in the short-wave channel becomes extremely crowded and there are many signals in the channel. The traditional digital down-conversion (DDC) channelized structure requires a lot of computation, mentioned in literature [2] of a highly efficient channelization structure can reduce the computational complexity of the system but with the emergence of new shortwave agreement, rate is rapid ascension of shortwave signal channel distribution, as part of the signal bandwidth can be up to $24 \mathrm{KHZ}$ is greater than that of ordinary narrowband receiver receive bandwidth, literature [2] channelized filter after the son signal may be caused by the truncation quilt channel across the channel signal distortion, affect the subsequent parameters estimation and decomposition reconstruction [3]. Literature [4] proposed a non-uniform channelization filtering method based on a modulation filter bank. This method needs to

\footnotetext{
* Corresponding author: ggkx@163.com
} 
predict the bandwidth and position distribution of the signal. However, in a multi-standard communication system, each sub-band signal in the intermediate frequency (IF) bandwidth is sometimes dynamic changes occur in the receiving process, so the method in [4] cannot be directly applied to such situations. Literature [5] proposed a "smart" digital channelization structure. When the signal changes dynamically, there is no need to change the analysis filter bank, and the dynamic channelization processing of the signal can be completed as long as the energy detection is updated. However, the noise in the shortwave channel has uncertain factors such as time-varying and frequency-varying. The dual-threshold energy detection proposed in [5]; the Wisahart random matrix theory detection mentioned in [6]; the highorder cumulant and matched filtering signal detection methods mentioned in [7], have a strong detection efficiency only under white noise. A wideband signal detection method based on morphological filtering was proposed in literature [8,9]. The noise floor in [8] is the connection line at the lowest point of the power spectrum. This method is greatly affected by noise burrs, and the interpolation processing in this paper will lead to the insufficient smoothness of the estimation of noise floor. The algorithm of [9] will cause the estimated noise floor to contain sub-band signals, and there is no method for sub-band signal extraction in the literature. Therefore, this paper proposes a cross-channel reconfigurable multi-phase high-efficiency channelization method based on improved morphological processing. This method has lower computational complexity and higher detection probability under colored noise environment.

\section{Description of the process}

The wide-band IF signal which is converted to baseband by orthogonal down conversion after sampling, are coarsely filtered through a uniform filter bank. The power spectrum estimation of the wide-band signal is completed by the Welch algorithm, and then the power spectrum is subjected to improved morphological filtering to estimate the noise floor, and the modified spectrum is obtained through the top hat operation, and then the spectrum is smoothed by the secondary morphological filtering, and the smoothed power spectrum perform threshold detection to estimate the position and bandwidth of the signal, Finally, the sub-channels where the sub-band signals are located are combined to complete the crosschannel signal reconstruction

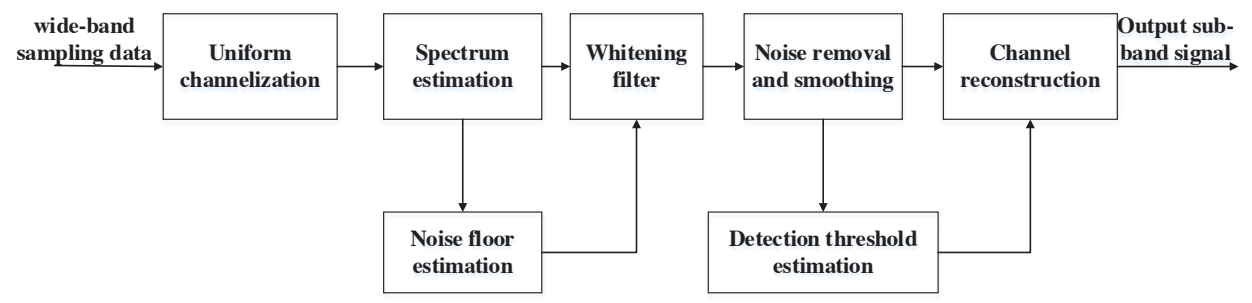

Fig. 1. Flow diagram.

\section{Principle of adaptive capture algorithm}

\subsection{Channelization principle of multi-phase filter bank based on DFT}

Assuming that the transmission channel is an additive Gaussian white noise channel, the received signal model is as follows: The concept of channelization is to divide the frequency band into several sub-channels through a set of filter banks covering the entire frequency 
band. Each filter is a channel output, and finally each sub-channel is moved to zero intermediate frequency through down conversion. This concept can be equivalent to moving the sub-band signal to zero intermediate frequency through down-conversion, and then passing through a low-pass filter with a bandwidth of the sub-band signal width. Due to the non-ideal characteristics of the low-pass filter, the transition band of the filter will cause a blind area for monitoring in the channel. In order to solve the channel blind zone problem; in order to meet the signal reconstruction conditions in this paper, the channel division method is shown in Figure 2.

Assume that there are a total of k sub-channels, and the center frequency of the k-th subchannel is

$$
w_{k}=\left(k-\frac{M}{2}\right) \cdot \frac{2 \pi}{M}, k=0,1, \ldots M-1
$$

where $w_{k}$ is the mixing angle frequency of the k-th channel, and $M$ is the total number of divided channels. The channel is divided in an even-type structure, and the filter banks overlap at the $3 \mathrm{~dB}$ bandwidth.

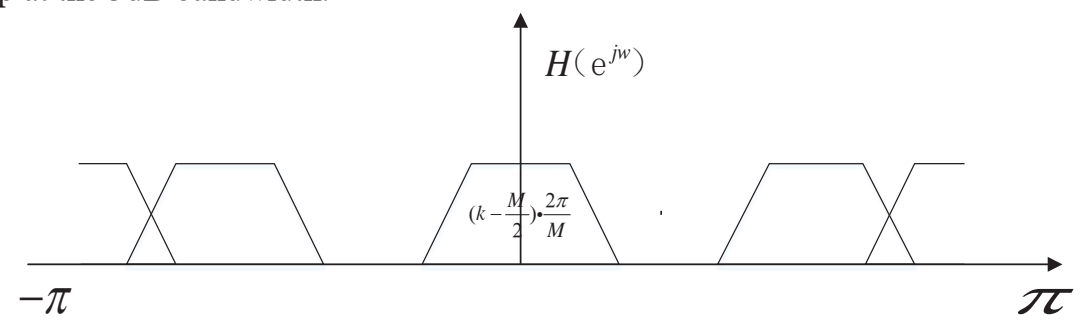

Fig. 2. Channel division method.

When the channel is divided by overlapping, if the maximum decimation is used to reduce the data rate, spectrum aliasing will affect the output of the sub-band signal. Therefore, extraction is performed by a decimation factor of $D=M / 2$ to avoid spectrum aliasing. In the traditional channelized structure, all frequency shifting and filtering operations are performed before extraction, which is bound to be unfavorable for real-time signal transmission. The multi-phase filter channelization structure of Fig. 3 can reduce the computational complexity of the system. The structure uses the multi-phase filter technology in the multi-rate signal processing theory to place the FIR filter at the end of the low sampling rate. The multi-phase decomposition of the filter is expressed as:

$$
H(z)=\sum_{d=0}^{M-1} z^{-d}\left[\sum_{n=0}^{L-1} h(n M+d)\left(z^{M}\right)^{-n}\right]
$$

The output of the kth channel after channelization is

$$
\begin{gathered}
\left.y_{k}(n)=(-1)^{d \cdot n} * D F T\left\{\left[x_{d}(n) * h_{d}(n)\right]\right\}(-1)^{d}\right\} \\
x_{d}(n)=x(n D-d) \\
h_{d}(n)=\left\{\begin{array}{ll}
h(D n+d), n=\frac{M}{D} * P, P \text { is an integer } \\
0, & \text { other }
\end{array}\right\}
\end{gathered}
$$

where $x_{d}(n)$ and $h_{d}(n)$ are the multi-phase components after the signal and the filter are extracted respectively. The multi-phase structure channelization reduces the computational complexity of the system; reduces the sampling rate of subsequent signals; and speeds up signal processing. 


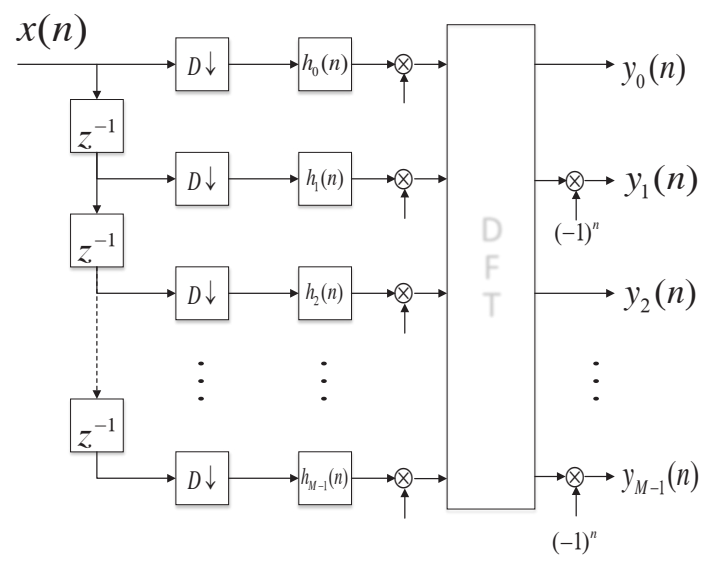

Fig. 3. Multi-phase channelized reception model.

\subsection{Channel reconstruction}

The analysis filter bank designed with the minimum interval $G_{\min }$ between signals divides the channel into $M$ sub-channels evenly. Since the bandwidths of the sub-band signals are not the same and part of the sub-band signals will appear in several adjacent sub-channels, it is necessary to perform adjacent channel combination to complete non-uniform channelization. In order to complete the accurate reconstruction of the sub-signal, the system i-th input signal $x_{i}(n T)$ and output signal $\widehat{x}_{i}(n T)$ satisfy the relationship shown in equation (3-6)

$$
\widehat{x}_{i}(n T)=c x\left[\left(n-n_{0}\right) T\right]
$$

where $c$ and $n_{0}$ are constants. The input signal and output signal can only have group delay and amplitude changes. The number of sub-channels is $M$, the fluctuation in the passband is $\delta$, and the prototype filter $h(n)$ should satisfy

$$
1-\delta \leq\left|H\left(e^{j w}\right)\right|^{2}+\left|H_{0}\left(e^{j(w-\pi / M}\right)\right|^{2} \leq 1+\delta
$$

In this paper, the position and bandwidth of the sub-band signal are determined by wideband power spectrum detection, and the state of the sub-channel that contains the signal is marked as "1", and the state of the sub-channel that does not contain the signal is marked as " 0 ". Assuming that the output sub-channel status is shown in Figure 4, sub-channels 1 3 and 5 6 should be combined and output. According to the method of literature [5] to complete the reconstruction and extraction of the sub-band signal, assuming that the i-th subband signal $x_{i}(n)$ occupies a total of $M_{i}$ sub-channels, define

$$
\widehat{M}_{i}=2^{\left\lceil\log _{2} M_{i}\right\rceil-1}
$$

Due to the requirement of anti-aliasing, the input data is extracted according to $D=M / 2$ method. In order to match the data rate of the input signal, the filter coefficients extracted by $M$ times should be interpolated with 0 . The multi-phase component of the reconstruction filter of the sub-band signal $x_{i}(n)$ can be expressed as

$$
F_{q}(z)=\sum_{r=0}^{m-1} h\left(q \frac{D}{\widehat{M}_{i}}+M r\right) z^{-2 r}
$$

where $q=0,1, \ldots, \widehat{M}_{i}-1$. The multi-phase component of the reconstruction filter in formula (3-9) is also the multi-phase component of the prototype filter. As long as the number of channels occupied by the sub-band signal is known, the corresponding reconstruction filter 
can be obtained from the prototype filter. The multi-phase structure extracted by crosschannel signal reconstruction is shown in Figure 5.

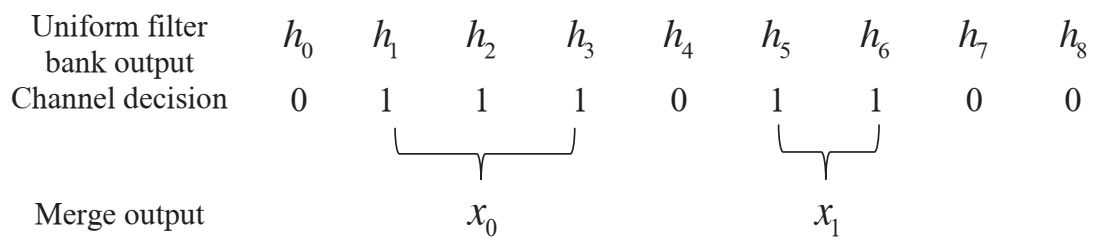

Fig. 4. Schematic diagram of uniform filter bank output merge.

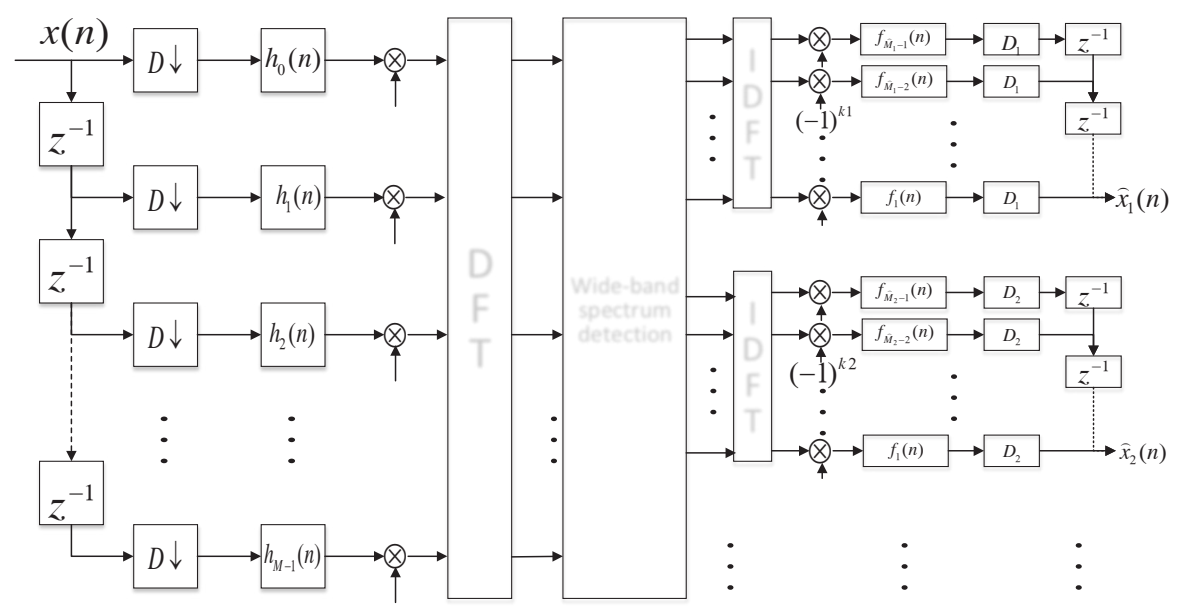

Fig. 5. Cross-channel signal reconstruction model.

\section{Noise floor estimation}

\subsection{Morphological filtering}

One-dimensional digital signal morphological operation is based on random set theory, which is a nonlinear transformation that locally modifies signal characteristics [10]. In some cases, the transformation of signals will distort their collective characteristics, and by selecting appropriate morphological operations and structural elements for processing, the morphological information of one-dimensional signals can be preserved. Assuming that $f(n)$ is a one-dimensional discrete signal defined on $F=\{0,1, \ldots, N\}$, and $g(n)$ is a one-dimensional discrete data defined on $G=\{0,1, \ldots, M\}, M<N$.If $f(n)$ is the input spectrum data and $g(n)$ is the structural element, then the four basic morphologies of morphology are transformed into

$$
\begin{gathered}
(f \Theta g)(n)=\min \{f(n+m)-g(m)\}(n=0,1, \ldots, N-M) \\
(f \oplus g)(n)=\max \{f(n-m)+g(m)\}(n=0,1, \ldots, N+M-2) \\
f \bullet g=(f \oplus g) \Theta g
\end{gathered}
$$




$$
f \circ g=(f \Theta g) \oplus g
$$

The above four formulas are morphological corrosion, expansion, closing, and opening operations. The expansion operation will reduce the valley value of the signal and expand the local value; the erosion operation will reduce the peak expansion valley of the signal. The open operation can smooth the peak of the signal; the closed operation can smooth the trough of the signal. The noise floor can be estimated by selecting appropriate structural elements and then using different combinations of four operations.

\subsection{Noise floor estimation algorithm}

In literature [8], the data is segmented by formula (4-5) and then the minimum value is extracted. Smaller structural elements are selected to perform morphological operations on the obtained minimum value data, and then data interpolation is performed to obtain an estimate of the noise floor. Perform whitening processing through top hat calculations. Since the noise floor obtained by this method is only the connection of the lowest point of the power spectrum of each segment, the estimated noise floor is not smooth enough and is greatly affected by noise burrs. Therefore, literature [9] proposed an improved algorithm (4-6), which eliminates the interference of signal peaks and valleys on the signal by performing open and closed operations on the original signal, and then add the results of the calculations. By averaging, the results obtained can better reflect the fluctuations of the noise floor. However, the closed operation in the improved method will cause the presence of sub-band signals in the estimated noise floor, and the peak value of the signal will be reduced during the top hat operation, which will have a greater impact on the signal detection probability and the corrected power spectrum will be affected by noise burrs When the SNR is low, the algorithm in [9] has a greater false alarm probability.

$$
\begin{gathered}
T_{\text {hat }}(f)=f-(f \circ g) \\
T_{\text {hat }}(f)=f-\frac{(f \circ g)+(f \bullet g)}{2}
\end{gathered}
$$

An improved algorithm to estimate the noise floor in this paper.

(1) The power spectrum estimation is carried out by Welch algorithm, which is to average the data segmented and windowed, which can effectively reduce the variance of the power spectrum estimation without seriously destroying the resolution. The modified power spectrum that divides the signal $x(n)$ of length $N$ into $p$ segments is

$$
P(\omega)=\frac{1}{P} \sum_{P=0}^{P} \frac{1}{M U}\left|\sum_{n=0}^{M-1} x^{p}(n) w(n) e^{-j w n}\right|^{2}
$$

where $x^{p}(n)$ is the p-th segment data, $w(n)$ is the added window function, and $U=\frac{1}{M} \sum_{i=0}^{M-1} w^{2}(n)$ is the normalization factor

(2) Select appropriate structural elements according to the method of literature [11], and the selection process of structural elements is shown in Figure 6. By formula (4-8), the noise floor of power spectrum is obtained by the mixed Morphological operation of the opening and closing, which better reflects the fluctuation of the real noise floor and solves the problem of removing the part of the signal amplitude as noise when performing the top hat operation in the literature [9]. Finally, a combination of opening and closing operations is performed for secondary smoothing to solve the problem of high false alarm probability caused by noise burrs when the signal-to-noise ratio is low and the problem of misjudgment of multi-carrier signals as multiple signals during threshold judgment. 


$$
\begin{gathered}
T_{1}(f)=\left[f-\frac{(f \circ g) \bullet g+(f \bullet g) \circ g}{2}\right] \\
T_{h a t}(f)=T_{1}(f)-T_{1}(f) \bullet g \circ g
\end{gathered}
$$

The noise burr of the corrected power spectrum in this paper is basically removed, and the noise power $\lambda \approx 0 d b$. On this basis, a certain offset can be added as the decision threshold. The value of the offset generally depends on the false alarm probability.

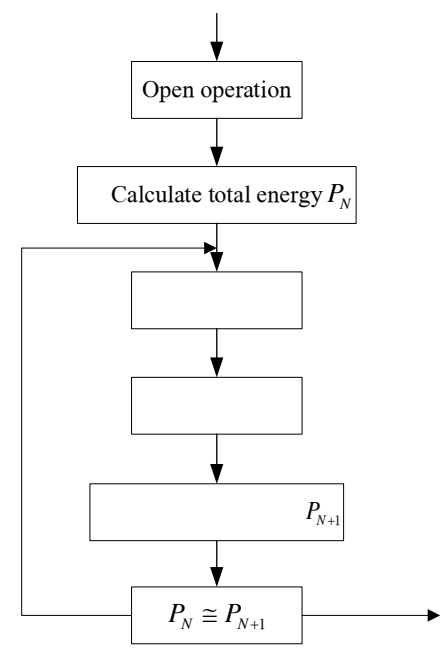

Fig. 6. The algorithm that selects the length of structural elements

\section{Simulation and analysis}

Assume that the wide-band signal to be processed is a $1 \mathrm{MHz}$ complex signal, and the sampling rate of the system is $1 \mathrm{MHz}$. The noise in the channel is the Gaussian white noise with variance $\sigma=1$. Suppose the bandwidth contains three signals, two linear frequency modulation (LFM) signals and a single frequency signal. The two LFM signals, in which the bandwidth of signal a is $20 \mathrm{KHz}$, the center frequency is $110 \mathrm{KHz}$, the bandwidth of signal $\mathrm{b}$ is $30 \mathrm{KHz}$, the center frequency is $215 \mathrm{KHz}$, and the center frequency of single-frequency signal $\mathrm{c}$ is $800 \mathrm{KHz}$. The spectrum diagram and sub-channel position of the wide-band signal are shown in Figure 7.

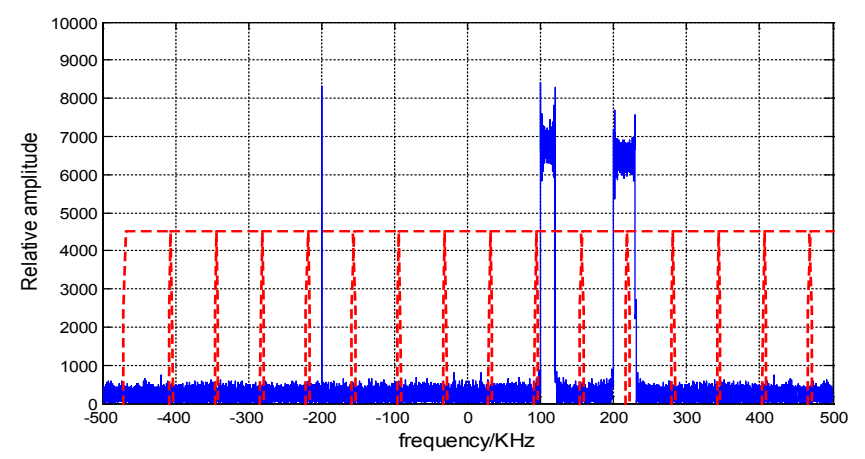

Fig. 7. Frequency domain waveform of the input signal.

In order to approximately satisfy (3-7), this paper uses the Parks-McClellan method to 
design the optimal prototype FIR filter. The FIR filter order is 1024, the stopband attenuation is $60 \mathrm{~dB}$, the $3 \mathrm{~dB}$ cutoff frequency is $31.25 \mathrm{KHz}$ and the filter banks overlap here. The number of sub-bands is 16 and the new arrivals are roughly divided in the manner shown in Figure 1. It can be seen from Figure 7 that the c signal is on the 5th channel; the $a$ signal is on the 10 channel; and the $b$ signal is across the 11 and 12 channels. The sub-band output after channelization is shown in Figure 2. From the simulation results, it can be known that the results after channelization are consistent with the actual situation.
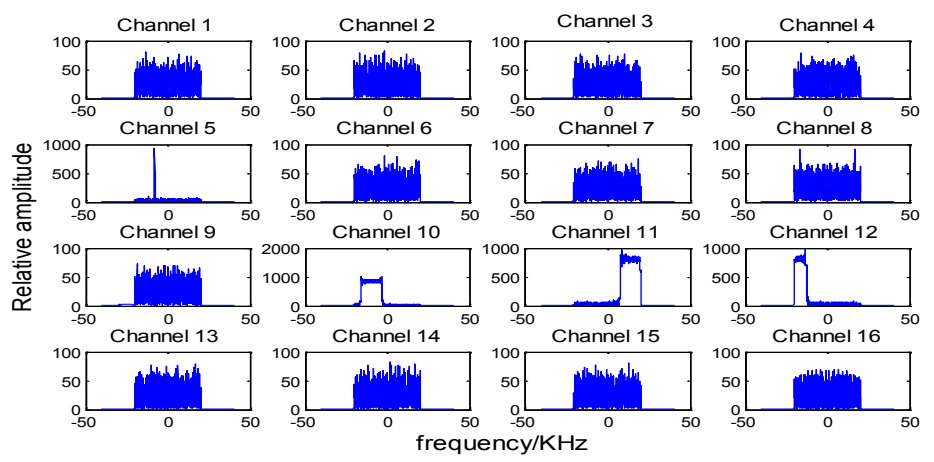

Fig. 8. The frequency domain waveform output of the sub-channel.

After the rough division of the channel is completed, a slow-changing noise floor with a maximum fluctuation of $20 \mathrm{~dB}$ is artificially added. Figure $5(a)$ is the power spectrum obtained directly, and Figure $5(b)$ is the spectrum obtained by welch spectrum estimation. It can be seen that the spectrum estimated by the welch algorithm is smoother.
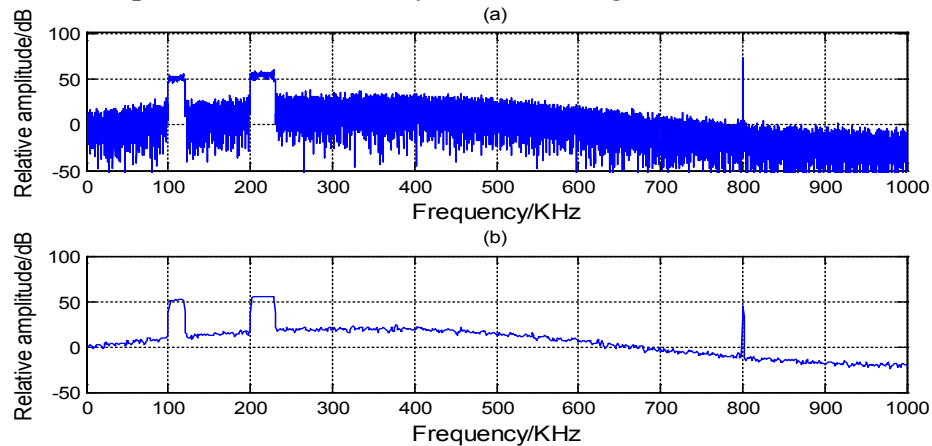

Fig. 9. Power spectrum after adding noise floor.

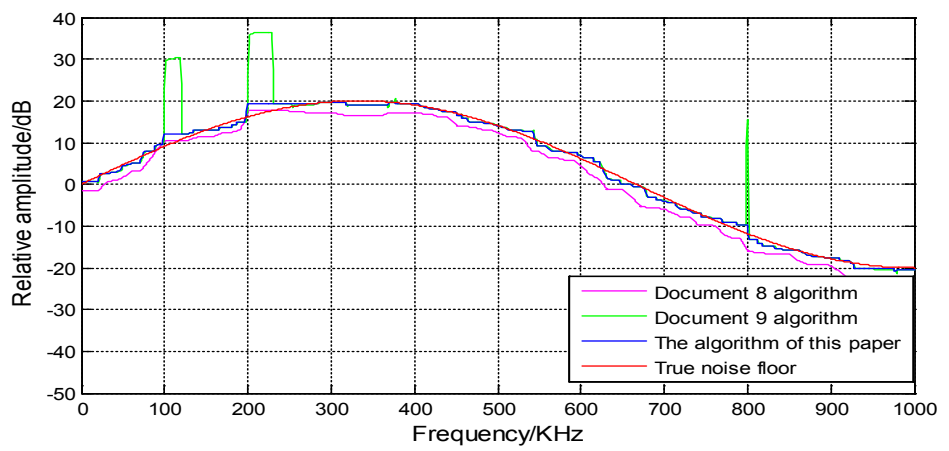

Fig. 10. Comparison of noise floor calculated by different algorithms. 
It can be seen from Figure 10 that the noise floor in literature [8] has a certain offset due to the influence of noise burrs and interpolation. The noise floor in literature [9] contains subband signal components, and the noise floor obtained by the algorithm in this paper is more Close to the actual noise floor.

It can be seen from Figure 11(b) that the noise burr of the power spectrum after the second smoothing is basically removed and has no effect on the signal amplitude. By changing the noise power to determine whether the system meets the approximate constant false alarm detection, 300 Monte Carlo simulations are performed under different noise powers to determine the false alarm probability under the constant judgment threshold $\eta=2$. It can be seen from figure 8 that the algorithm approximately satisfies CFAR detection.

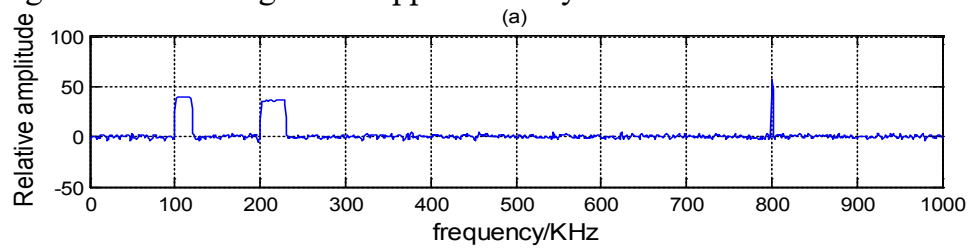

(b)

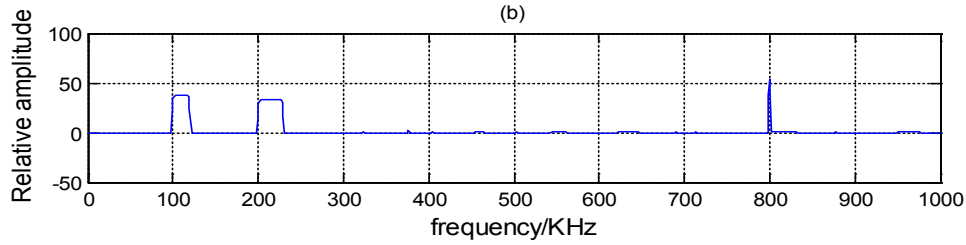

Fig. 11. (a)Power spectrum after removing the noise floor.(b)Power spectrum after secondary smoothing.

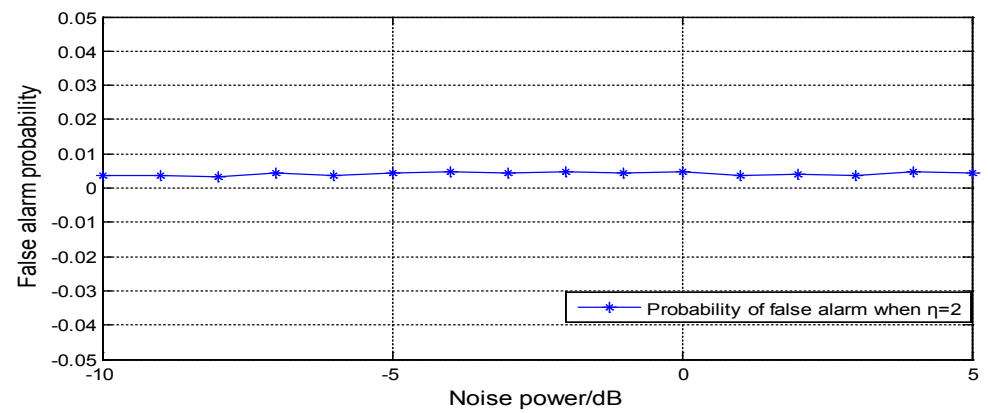

Fig. 12. False alarm probability under different noise power.

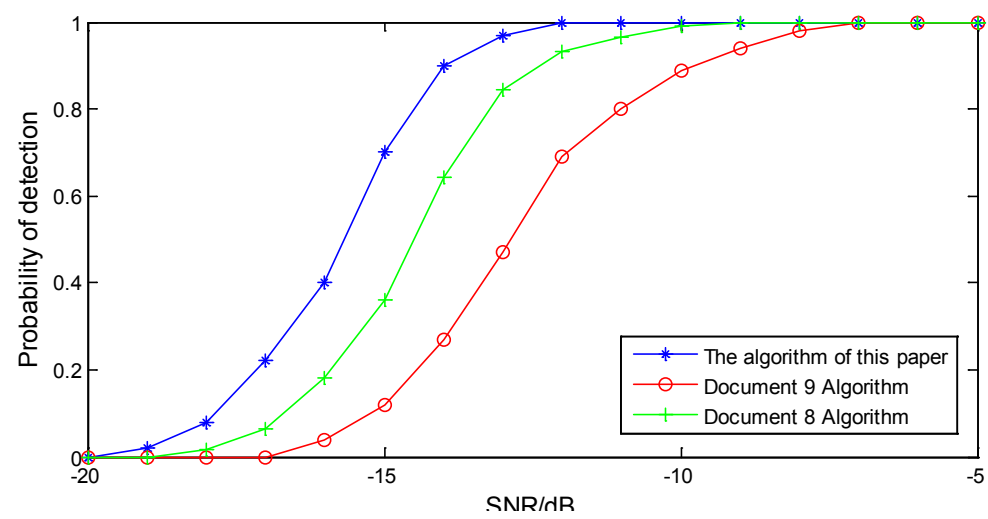

Fig. 13. Comparison of detection performance under different SNR. 
When the false alarm probability is about $10^{-4}$, this paper performs 300 Monte Carlo simulations on the $30 \mathrm{KHz}$ LFM signal under different SNR ratios. It can be seen from Figure 13 that the detection probability is still more than $90 \%$ when the SNR is $-14 \mathrm{~dB}$. Detecting the peak of the power spectrum after the correction, it is detected that the signal $b$ is in the 11 and 12 channels, and there is a cross-channel situation, so the two channels must be combined to complete the sub-signal reconstruction.

Reconstruct the signal according to section 3.2. Figure 12 shows the signal spectrum after reconstruction. It can be seen from Fig. 12 that the prototype filter obtained by the ParksMcClella algorithm has almost no distortion in the transition band during channel combination, and the obtained signal can meet the approximate reconstruction conditions.

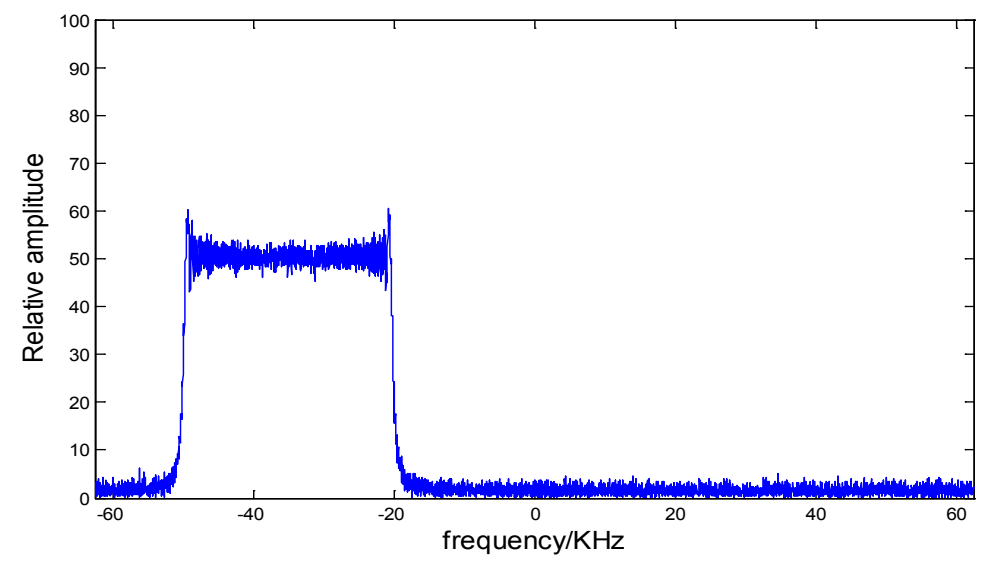

Fig. 14. Signal reconstruction frequency domain waveform.

Complexity analysis: The complexity of multi-phase reconfigurable channelization is compared with that of conventional DDC structures. Suppose the number of channels is $M=128$, the number of signals is $N$ and the order of the prototype filter is $L=1024$. When the sampling rate is $f_{s}$, the output of the sub-channel is multiplied by the sampling rate of the output sampling points in order to count the computes at different sampling rates to the same time scale. The calculation amount of complex multiplication of the two structures is:

a. Non-uniform channelized receiver based on DDC:

$$
a=f_{s} \cdot N+L \cdot \sum_{i=1}^{N} \frac{f_{s}}{M} \cdot \hat{M}_{i}
$$

b. Reconstructed channelized receiver based on multi-phase filter bank:

$$
\begin{gathered}
b_{1}=\frac{f_{s}}{D}\left(\frac{M}{2} \cdot \log _{2} M\right)+\left(\left(1+\frac{L}{D}\right) \cdot M\right) \cdot \frac{f_{s}}{D} \\
b_{2}=\sum_{i=1}^{N}\left(\left(\left(1+\frac{L}{D}\right) \cdot \widehat{M}_{i}\right) \cdot \frac{f_{s}}{D}+\left(\frac{\hat{M}_{i}}{2} \cdot \log _{2} \widehat{M}_{i}\right) \cdot \frac{f_{s}}{D}\right) \\
b=b_{1}+b_{2}
\end{gathered}
$$

The calculation amount of the two methods is shown in Table 1. Compared with the nonuniform signal of the traditional DDC structure, as the number of signals increases, the computational complexity of the reconstructed channelized structure based on the multiphase filter bank is significantly lower. 
Table 1. Complex number multiplication times of two structures.

\begin{tabular}{|c|l|l|l|}
\hline \multirow{2}{*}{ Number of sub-signals } & \multicolumn{1}{|c|}{$\hat{M}_{i}=2$} & \multicolumn{1}{|c|}{$\hat{M}_{i}=4$} & \multicolumn{1}{|c|}{$\hat{M}_{i}=8$} \\
\hline \multirow{2}{*}{$N=1$} & $a=17 f_{s}$ & $a=33 f_{s}$ & $a=65 f_{s}$ \\
\cline { 2 - 5 } & $b=40.01 f_{s}$ & $b=43.06 f_{s}$ & $b=55.19 f_{s}$ \\
\hline \multirow{2}{*}{$N=5$} & $a=85 f_{s}$ & $a=165 f_{s}$ & $a=325 f_{s}$ \\
\cline { 2 - 5 } & $b=42.08 f_{s}$ & $b=59.3 f_{s}$ & $b=119.95 f_{s}$ \\
\hline \multirow{2}{*}{$N=10$} & $a=165 f_{s}$ & $a=330 f_{s}$ & $a=650 f_{s}$ \\
\cline { 2 - 5 } & $b=49.16 f_{s}$ & $b=79.6 f_{s}$ & $b=200.9 f_{s}$ \\
\hline
\end{tabular}

\section{Conclusion}

Aiming at the computational complexity of the traditional DDC non-uniform channelization structure and the influence of noise uncertainty on the detection probability, this paper proposes a multi-phase reconfigurable channelization structure based on morphological operations. Firstly, the channel is uniformly and roughly divided, and then the broadband power spectrum is processed by the morphological processing to determine the bandwidth and position of the signal, and finally the cross-channel signal is reconstructed by the reconstruction filter bank. This paper analyzes from two aspects of computational complexity and detection probability. The results show that this paper has lower complexity compared with the traditional DDC non-uniform channelized structure. It can achieve nearly constant false alarm detection in a color noise environment, and the detection probability of LFM signals can still reach more than $90 \%$ at a SNR of $-14 \mathrm{~dB}$.

This work was supported by the National Natural Science Foundation of China under Grant U1736107

\section{References}

1. Yong zheng Lv. Study on Mil-STD-188-110C Waveform Detection Algorithm under short-wave Broadband condition[D]. South China University of Technology.(2019)

2. chao Li. Research on Channel Digitization of Wireless Receiver and FPGA Implementation[D]. University of Electronic Science and Technology of China.(2018)

3. Yang Wang, Zhongwei Xu, Siyi Cheng. Tolerance and parameter estimation combine to solve the cross-channel problem of channelized receivers[J]. Journal of Air Force Engineering University (Natural Science Edition). 16(02),61-66. (2015)

4. W. A. Abu-Al-Saud and G. L. Stuber.M Efficient wideband channelizer for software radio systems using modulated PR filterbanks. IEEE Transactions on Signal Processing.52,10.(2004)

5. Nan Zhang,Weixiang Lv. Wide-band signal digital channelization and crosschannel reconstruction detection technology[J]. Telecommunications technology. 57(02),191-196.(2017)

6. U. Y. Mohamad and D. Dahlhaus.Cognitive RAdio sensing based on joint distribution of pseudo WIShart matrix Eigenvalues. Wireless Telecommunications Symposium. Washington, DC.1-6.(2014)

7. Peng Li.Gang Chen.Kui Zhang. New signal detection method based on high-order cumulant and matched filtering[J]. System Engineering and Electronic Technology.2006(01).31-33.2006 
8. Yongming Wang.Eryang Zhang.Qiaojin Cheng. An effective broadband digital reconnaissance[J]. Signal processing.26(2),208-212.(2010)

9. Chao Zhang.Hong Ma.Yiwen Jiao. Dynamic channelization subband signal detection method based on morphological operation[J]. Electronic measurement technology.41(23),44-48.(2018)

10. Shu Liu. Research on the Application of Mathematical Morphology in Signal Processing[D]. Dalian University of Technology.(2006)

11. Tianli Jiang.Hua Peng.Kexian Gong. Wideband signal detection method under multi-scale morphological filtering[J]. Signal processing.30(09),1055-1063.(2014) 